\title{
Pperation of the TR30 "Industrial" Cyclotron
}

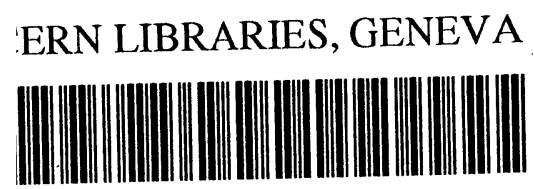

CM-P00068387

\author{
Karl Erdman, R. Dawson \\ Ebco Technologies, TRIUMF
}

B. Milton, N. Stevenson

TRIUMF, 4004 Wesbrook Mall, Vancouver, B.C. V6T 2A3

\section{Abstract}

The $30 \mathrm{MeV}$ TR30 cyclotron installed for Nordion bas been in operation for two years. The use of industrial process controllers, the external $\mathrm{H}^{-}$ion source and cryogenic pumping has made this machine easy to operate and reliable with extracted beams of $500 \mu \mathrm{A}$. The strong focussing properties of the magnet and low internal beam losses have reduced residual radiation fields so that there is negligible exposure to the operators during machine servicing. The main characteristics and features of the machine leading to a $98 \%$ accelerator availability are discussed in this paper. As well as this the extensions to the cyclotron allowing the acceleration of deuterons to $15 \mathrm{MeV}$ are outlined.

\section{INTRODUCTION}

The TR30 cyclotron was designed to be an accelerator for the commercial production of isotopes and as such to be a highly reliable, low maintenance, automatically operated, and low residual activity system [1].

The basic design is for a compact magnet system with strong focussing of the particles to avoid losses during the acceleration process and using negative ions to give almost $100 \%$ extraction efficiency of the beam. The losses in the cyclotron are further minimized by the use of cryopumps to produce the vacuum in both the injection system and in the cyclotron itself. This reduces the losses due to stripping of the negative ions in the vacuum tank by eliminating residual contamination due to the presence of heavy molecules from the backstreaming of oil from the diffusion pumps.

The need to vent the cyclotron during servicing is obviated by the use of valves on both the ion source injection line and the stripper foil carriage mechanism so that routine maintenance can be carried out while maintaining a good vacuum in the tank. The life of the cryopumps is extended by this process and the "hard" vacuum established in the tank simplifies the operation of the rf system as well as improving the beam transmission.

\section{OPERATION}

Values extracted from a computer log record of the operating values of the various components of the system, while delivering two simultaneous beams down two external beam lines, are assembled in Table 1 .

These values are taken from the hard copy output of the control system [2] which also continuously monitors and displays the input and output parameters of all of the system components on the color graphic display screens as well as alpha numeric output. System set points can be altered by using a mouse pointer or by typing in new values by means of the computer keyboard. The control software is highly flexible and permits the operators to define macros which have greatly aided in the "turn on" and tuning procedures as well as in maintaining system stability.

Table 1 System parameters during dual beam operation.

\begin{tabular}{lc}
\hline \hline RF power & $35 \mathrm{~kW}$ \\
Main magnet power & $33 \mathrm{~kW}$ \\
Beam current $(29 \mathrm{MeV})$ & $2 \times 220 \mu \mathrm{A}$ \\
Extracted current & $500 \mu \mathrm{A}$ \\
Target collimator current & $25 \mu \mathrm{A}$ \\
Ion source arc current & $15-20 \mathrm{~A}$ \\
Ion source arc power & $\sim 2 \mathrm{~kW}$ \\
Tank vacuum & $3 \times 10^{-7}$ torr \\
Total TR30 Facility (inc. processing & $175 \mathrm{~kW}$
\end{tabular}

areas and bldg. services) Power con-

sumption

The target system has been developed by NORDION to permit the operation of the system automatically by the use of the control computer and uses the same industrial control hardware and software that is used to control the cyclotron. Isotopes are transferred from the position of the irradiation to the processing hot cells by the use of a pneumatic transfer system [3]. The system has been developed over the course of the last 10 years of isotope production by NORDION and the performance of the system is shown in Fig. 1 where the production as a function of time from the commissioning of the TR30 cyclotron is displayed.

The servicing of the cyclotron is carried out in two different procedures. The first is the regular maintenance in

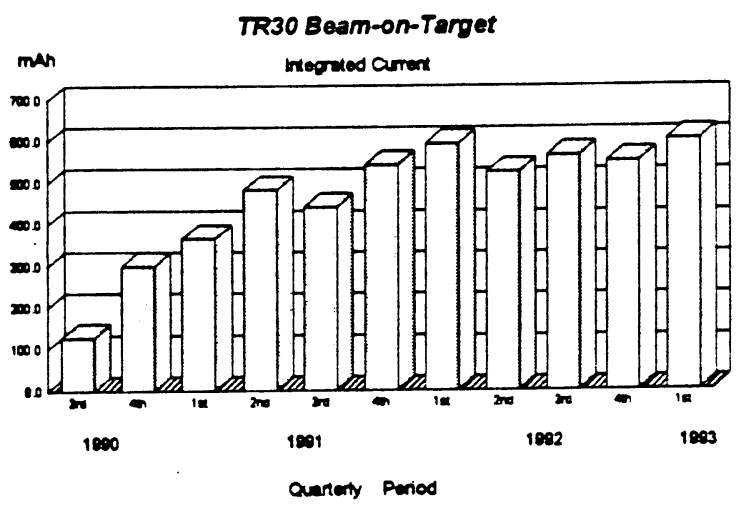

Fig. 1. Beam delivery by quarter. 
which the tank is not vented and involves the ion source filament and the stripping foil carousel. The ion source filament is normally replaced once every 3 weeks. The duration of the operation is about 4 hours. The performance of the ion source is logged by the control system and the state of the filament is determined by the filament power required to produce a given output current from the system. The life of the stripping foil is between 20 and 50 $\mathrm{mA}$ hours of extracted beam. The multifoil cartridge is changed during a filament replacement when it is deemed necessary to have a spare foil ready for operation.

The second procedure is the regular yearly preventative maintenance in which the tank is vented and the state of the inflector and the injection optic elements are inspected as well as other parts of the system which are impacted by the beam. The dc beam injected during the operation of the system at high current is $5-6 \mathrm{~mA}$ and erosion of the lenses occurs under the high current conditions. This service normally requires one 8 hour shift. If it is deemed necessary the cryopumps are regenerated during this service period.

A graph of the availability of the cyclotron and of the various system failures is given in Fig. 2. It can be seen that the main problems associated with the system failures are associated with the high power targets that are being irradiated. As development of the targets continues the total availability of the system is expected to increase. A comparison of the radiation exposure of the operators per unit of charge delivered to the targets between the CP42 and TR30 operated by the same team is given in Fig. 3. The dramatic reduction is due to the decrease in beam

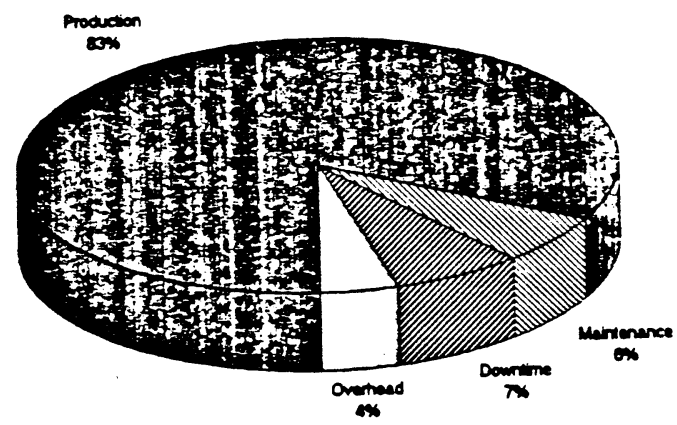

Fig. 2. TR30 operation system availability for 1993.

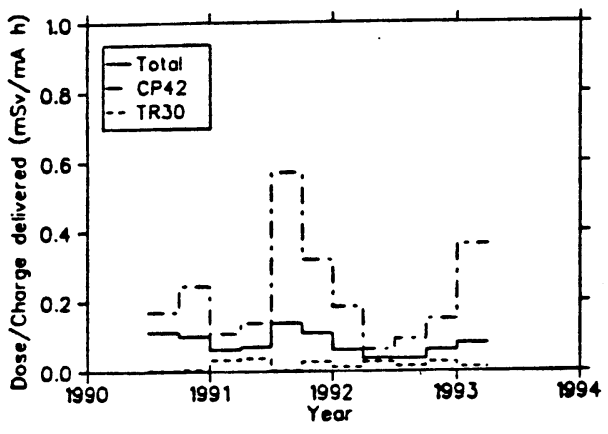

Fig. 3. Radiation levels in CP42 (solid line) and TR30 (dashed line). losses in the cyclotron with the external ion source and the clean vacuum in the tank.

\section{DEUTERON OPERATION}

To permit the operation of the cyclotron for the acceleration of deuterons, trim coils were added in the valleys of the magnet Fig. 4. The tuning stems on the resonators were extended by the use of a coaxial switch [4] to allow the if system to be tuned to the $4^{\text {th }}$ harmonic of the rotation frequency of the deuterons. By accelerating both the protons and the deuterons in the $4^{\text {th }}$ harmonic mode the tuning of the cyclotron for protons and deuterons is greatly simplified. The magnet is shimmed for protons and isochronizing coils are only used during the deuteron operation. The if voltage on the resonators is reduced by a factor of two so that the deuterons follow exactly the same orbits in the cyclotron as the protons but at $1 / 2$ of the energy. The energy of the injected beam is also reduced to $1 / 2$ of the proton energy. The maximum extracted deuteron beam current can be as much as $70 \%$ of the proton current using a common ion source. This cyclotron can be used as an extremely prolific source of neutrons for industrial neutron radiography as well as a deuteron accelerator for isotope production.

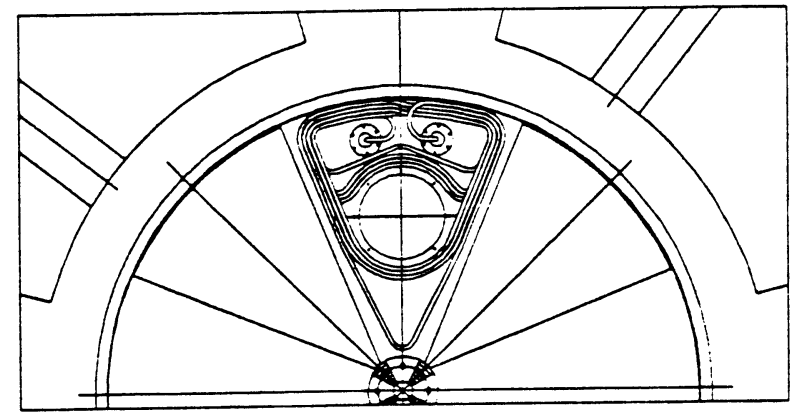

Fig. 4. Trim coils for deuteron operation.

\section{REFERENCES}

[1] B.F. Milton, et al. "First beam in a new compact intense $30 \mathrm{MeV}$ H- cyclotron for isotope production", Proc. of the European Particle Acceleration Conference, Nice (1990) p. 1812.

[2] R. Keitel, et al., "The TR30 control system - a case for off-the-shelf hardware", Proc. of the $19^{\text {th }}$ International Conf. on Cyclotrons and their Applications, Vancouver, B.C. (World Scientific, Singapore, 1992) p. 685 .

[3] J.J. Burgerjon, et al., "A high power target system for radioisotope production" Proc. of the $11^{\text {th }}$ International Conf. on Cyclotrons and their Applications, Tokyo (1986) p. 634.

[4] M. Eiche, et al., "Dual frequency resonator system for a compact cyclotron" Proc. of the $19^{\text {th }}$ International Conf. on Cyclotrons and their Applications, Vancouver, B.C. (World Scientific, Singapore, 1992) p. 515. 